\title{
Building an On-ramp for the Internet of Things
}

\author{
Scott Jenson, Roy Want, Bill N. Schilit \\ Google Inc. \\ 1600 Amphitheatre Parkway \\ Mountain View, California, USA \\ \{scottj, roywant, schilit\}@google.com
}

\author{
Robin Kravets \\ University of Illinois, Urbana Champaign \\ Department of Computer Science \\ 1304 W. Springfield Ave, Urbana, Illinois 61801 \\ rhk@illinois.edu
}

\begin{abstract}
The Internet of Things (IoT) is in its infancy, similar to the state of the Internet before the World Wide Web made it an indispensable tool for communication, business and entertainment. A similar revolution is needed for the IoT to become really useful. This paper identifies some important problems with today's IoT involving discovery and control, and proposes some solutions based on a concept we call the Physical $W e b$, an integration of the IoT with Web technologies.
\end{abstract}

\section{Categories and Subject Descriptors}

H.5.2 [Information Interfaces and Presentation]: User Interfaces-Input devices and strategies.

\section{General Terms}

Management, Design, Security, Human Factors, Standardization

\section{Keywords}

IoT; Physical Web; UriBeacon; Discovery; Bluetooth Low Energy

\section{INTRODUCTION}

The Internet of Things (IoT) is rapidly being integrated into our everyday lives. From connecting to a single smart device, such as a thermostat, to an ensemble of devices [1], such as the lighting system in an office building, we can interact with the environment around us. The availability of these devices is growing rapidly, with an estimate of 26 billion devices by 2020 [2]. However, interaction with so many diverse devices will quickly become intractable using the current model for native apps.

Whether dealing with a single device or an ensemble, there needs to be an easy-to-use interface to present to the user. Current solutions for interacting with IoT devices have relied on the use of smartphone apps. For use cases ranging from allowing a person to walk into a room and turn up the heat, to controlling the set of lights in a building, developers can design and implement a

Permission to make digital or hard copies of all or part of this work for personal or classroom use is granted without fee provided that copies are not made or distributed for profit or commercial advantage and that copies bear this notice and the full citation on the first page. Copyrights for components of this work owned by others than ACM must be honored. Abstracting with credit is permitted. To copy otherwise, or republish, to post on servers or to redistribute to lists, requires prior specific permission and/or a fee. Request permissions from permissions@acm.org.

IoT-Sys 2015, May 18, 2015, Florence, Italy.

Copyright 2015 ACM 978-1-4503-3502-7/15/05 ...\$15.00

http://dx.doi.org/10.1145/2753476.2753483 custom app and give the user control. Given the ubiquity and power of smartphones, building such apps is a good start in the right direction.

The trend, however, is that the number of smart devices and ensembles is growing, and along with this growth, there is a growing diversity of apps to interact with them. Furthermore, demand for IoT is moving beyond private and corporate spaces, and into the public world. People will be interacting with IoT devices in stores, airports and bus stops. While users may be willing to install relevant apps for their home or work spaces, which they interact with on a daily basis, installing an app for every IoT device they encounter during the day will quickly discourage them from ever using public IoT infrastructure. Instead, a lightweight solution where people could just walk up and use a device, without having to install any app, could overcome the hurdle of a 'difficult to use' IoT experience.

In the following sections we describe how IoT devices can be merged with Web technologies, and enable the traditional Web browser to become a single point of IoT interaction for users. We describe how every IoT device can have an associated Web page and broadcast a URL using standard RF protocols. Smartphones receiving multiple URLs can process them to produce a page-rank of nearby IoT devices using proximity as a guiding principle. IoT status monitoring and control is then achieved by direct interaction with the corresponding Web server.

\section{WEB BROWSING, SEARCH AND IoT}

To understand how to manage the massive increase in scale expected from the integration of IoT apps and devices, it is interesting to look back to how the scalability was handled in the early Internet and Web [3]. In the early 1990s the World-WideWeb was born on top of the Internet. Early solutions for managing scale were not effective, for example, single pages that attempted to catalog the entire Internet. It was quickly realized that a Web browser needed to integrate a search mechanism resulting in the familiar browser and search toolbar we use today. This scalable paradigm has become widely used and is the foundation of many Internet companies based on the Web today. As a result, the Web browser, plus search paradigm, is familiar to anybody that uses a networked computer.

The IoT is likely to increase the scale of the Internet as much as $100 \mathrm{x}$, and there is no reason to move away from the browser and search paradigm. However, today many IoT products enable action through proprietary solutions, which introduces several problems. First, the user needs to install a specific app on their mobile device. Second, the app's GUI may be unfamiliar to the user and non-intuitive to use (native apps often have more 
diversity than, for example, Web pages). Finally, installing an app for every device, or even an ensemble of devices, does not scale to the thousands of IoT devices that a user may encounter every day. To make the IoT intuitive to use and universally accessible to everybody, we believe a Web browser paradigm is a strong contender for the single user-facing app solution. To realize this solution, devices must be able to advertise a URL and the user's smartphone, or ensemble of wearable devices, must be able to manage the hundreds of device URLs that it may discover in any given environment. Furthermore, the Web is open, and isn't the product of any one company. The Web is a standard, and URLs can be resolved without a single company controlling the gateway (e.g. DNS [4]), making it possible for unencumbered innovation.

\section{PROXIMATE DISCOVERY}

The first step for effectively solving the 'IoT walk up and use' problem is to automatically identify devices, or things, that are nearby, and use these identifiers as a means to query and control them. There are many technologies that have been explored in earlier research, but all have limitations that make them unsuitable, e.g. RFID (due to a lack of mobile readers), QR codes (labor intensive, and can only identify one device at a time), GPS (only works outside, and lacks precision). However, the relatively new Bluetooth Low Energy (BLE v4.0) standard [5] provides a mechanism for each device to periodically emit an RF packet with an identifier that can be received by any modern smartphone since about 2011. The standard allows great flexibility in the information encoded in it, from UUIDs in devices known as iBeacons, to URLs in the UriBeacon open standard [6]. In the context of a Web-based IoT, UriBeacons can provide smartphones with a list of nearby IoT devices with associated Web pages and control functionality. We call this the Physical Web [7], which is the integration of the IoT with Web technology.

UriBeacons signal the presence of nearby IoT devices up to 10 meters away; therefore a smartphone in a smart environment might receive large numbers of UriBeacon packets. A practical system could filter this list, if it could estimate the distance of each device using RF ranging.

When working with a smartphone, one of the most common approaches to determine range is to subtract the received signal strength (RSSI) from the originating transmit power to yield pathloss, and then translate path-loss into a distance estimate. In an ideal world, this would be a simple translation, since the wireless signals follow well-known physical rules. Essentially, the strength of a transmitted signal roughly attenuates with the inverse square of the distance. Unfortunately, we do not live in a vacuum, transmit antennas are not point sources, and wireless signals experience multipath interference in a building, all making it difficult to directly translate path-loss to distance. In the end, for an app that requires precise ranging information, path-loss has limited accuracy.

However, despite these challenges there is a trend between pathloss and range. Assuming a transmit power of about $0 \mathrm{dBm}$, a strong correlation between path-loss and range exists for small distances on the order of $0.5 \mathrm{~m}$. The correlation gets noisier between $0.5 \mathrm{~m}$ and $2 \mathrm{~m}$, and very noisy beyond $2 \mathrm{~m}$. Given this finding, validated experimentally, we can use the path-loss parameter as a rule of thumb to determine whether a UriBeacon is in one of four defined regions: Nearest, Near, Mid, and Far. For the purposes of a Physical Web browser application, this differentiation is sufficient.
Although Wi-Fi could be used instead of Bluetooth LE in some situations, many Wi-Fi networks are secured with a passcode and are not accessible to mobile devices that happen across them. This is one reason Bluetooth LE broadcasts are so important; it allows devices to advertise information in a way that requires no prior association or pairing. Essentially, the advertisements are public broadcasts and can be received by all. By including the signal strength (Tx-Power) in these transmissions, any receiver can estimate the range/region of a remote device. This virtual identifier, formed from the combination of a URL and ranging data calculated using path-loss, makes it possible to build our Physical Web solution based on today's smartphones and existing standards. However, we would like to note that if a wireless local area network is available and accessible, multicast solutions [8] provide additional augmentation of the discovery mechanisms described here.

\section{EPHEMERAL INTERACTION}

Allowing each device or ensemble to offer up a modest Webpage is often met with skepticism because native smartphone apps are considered superior. However, most of these smart devices have very straightforward needs, such as a simple on/off switch, or the ability to scroll through a short list of information. The Web is perfectly fine for this. In addition, the Web is ultra-lightweight: walk up to a device, view its Web page and interact or move on. The entire interaction becomes ephemeral.

Such interaction turns the entire native application model on its head. Native apps are in effect caches of functionality that can be used over and over again. By using this ephemeral approach, the Physical Web assumes short, one-time Web-based interaction with a device, allowing the Web app to clean up after itself once the user has moved on. Of course, users can easily save a Webpage if they wish and new Web technologies like Service Worker [9] make this especially powerful. However, even if a user interacts with, for example, a particular bus-stop on route to work each day, this Web-like flow requires only a few finger taps and is not a heavy burden on the user experience.

Furthermore, we would argue that this switch to ephemeral interaction has a much bigger effect; it redefines what it means to be a smart device. If every device is capable of having a virtual flash card giving the user more information or interaction, it will change what it means for devices to be smart.

The Physical Web's primary value is to enable a device to place at users' fingertips anything from a tiny piece of location-based information, to a full-blown Web app.

As a result, devices or objects that may have been previously considered dumb now become smarter. If anyone can peek into an object's virtual flash card, it makes it nearly impossible for it to be lost. For example, luggage and pet collars can now offer up a phone number, movie posters can offer up previews and ticket sales, malls can offer kiosk style maps anywhere in the space. None of these devices are inherently smart, but they all allow for much smarter interaction.

Each of these examples, taken individually, is moderately useful. Taken as a whole, however, they imply a vast "long tail" where anything can offer information and utility.

\section{IoT SEARCH}

In practice, browsers in combination with search engines do more than provide us with a list of textual URLs, they also provide us 
with a useful and intuitive user interface with snippets of each page, filtered content, and rank ordering of the list. In a Physical Web browser we would expect similar features that are expanded on below:

Snippets: a URL is often a textually obscure reference to a Webpage and difficult for a user to interpret. In practice, it's better to pre-fetch data from broadcasted Websites in order to provide a user with not only the URL, but a title and snippet of the referenced Web page to guide users to the required information. For the Physical Web, this would be acheived using an additional proxy service. In the case of IoT devices, the snippet may contain identifiable information about the device, such as type, name, model, and its status. Simple graphical controls could be provided within the snippet to reduce interaction time e.g. up and down buttons to change a room's temperature setting.

Filtering: Search engines also protect us from nefarious Websites and reduce a user's exposure to spam sites that are designed to grab your attention when they don't actually contain the information you were looking for. In this case they serve as useful filters for the Web. The IoT is no different, and will soon be polluted by nefarious devices and services that are so common on the Web. Although the Physical Web browser is the user facing interface for UriBeacons, the information it shows can be preprocessed by a proxy service using a crowd-sourced database of nefarious device URLs, and can filter accordingly.

Ranking: Probably the most important user facing feature of a search engine is its ability to rank its search results based on relevance to the search term and the user. In other words, as a heuristic, the most relevant items are at the top of the list. As stated earlier, the IoT benefits from proximity ordering, which can be approximately determined by RF signal strength. However, this is not the only parameter that can be considered. Other parameters include related information that also exists on the Web e.g. related products, companies offering value added services, vendors of the device, companies offering replacement parts, history of prior IoT interactions, and the state of an IoT device e.g. a carbon monoxide sensor detecting a critical reading. The algorithms for ranking have always been interesting [10], and even controversial as companies vie for placement at the top of the rank list. For IoT there are more dimensions to consider, and the rank algorithm will be a subject of future research.

\section{SEMANTICS AND IoT CONTROL}

If the Physical Web understands the content of Webpages, then it can create rich snippets and quick actions for nearby things. Rich snippets are detailed information that can help users answer specific needs. For example, the snippet for an exercise machine at a gym might show the muscle groups and level of difficulty of use. These snippets help users understand when a thing is relevant to them. At the next level is Quick Actions that give users a path to interacting with the Internet of Things. For example, the quick action for a treadmill machine might be to share the user's weight, duration, and course preferences with the machine over Bluetooth, or a connected cloud service.

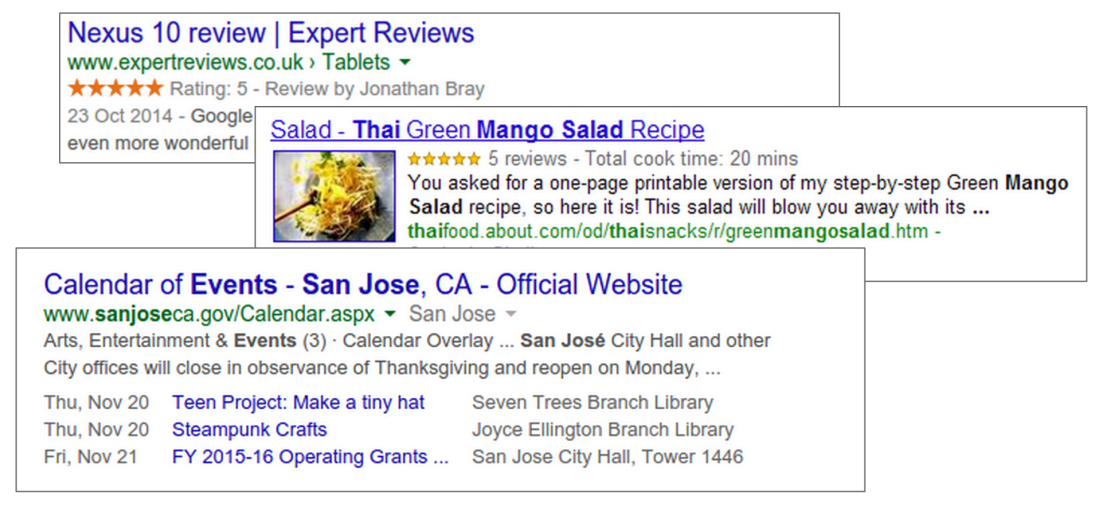

Figure 1: Structured Data for Web Snippets 

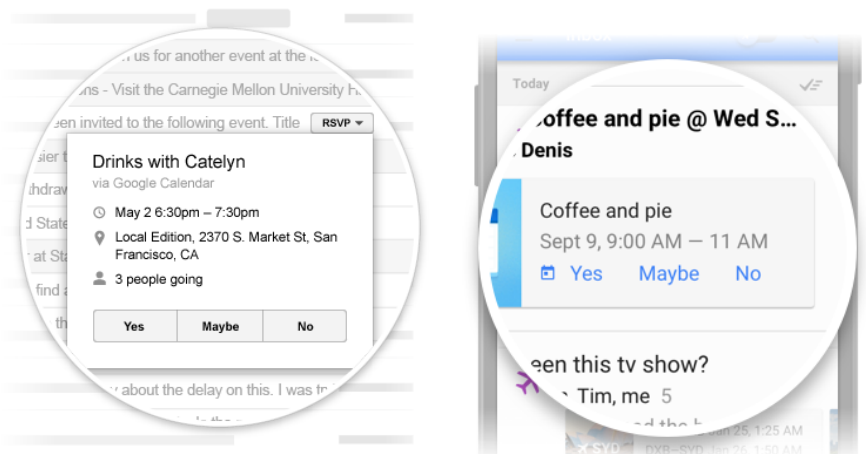

Figure 2: RSVP actions from Structured Data in Gmail

Both rich snippets and quick actions are enabled by structured data within the content of Webpages. Structured data for the Web is already well established [11], so we believe the use of structured data for the Physical Web should be relatively straightforward. In today's Web, major search engines use structured data for search results: to show recipe details, musician's songs, restaurant ratings (Figure 1). Structured data is also used in email to RSVP to a calendar invite, check into a flight, or view package delivery information (Figure 2).

These and other mechanisms use schema.org definitions. The schema is a common way for Webmasters and email senders to add information to HTML pages that can be understood by search engines and email programs.

Structured data within Webpages of IoT devices can also enable use cases at a machine-to-machine level. For example, by automatically dimming lights in a home as a person leaves the room. The rich description of a device and its interfaces can all be stored in Webpages. In some protocol stacks, such as Universal Plug and Play (UPnP) [12], the descriptions of devices, their control points, and events, are provided directly by UPnP-enabled devices. In our Physical Web vision, defining the Web as the intermediary for IoT has the advantage of connecting these devices to a vast array of existing Web-based tools and services.

\section{CONCLUSION}

The Internet of Things needs the ability for people to just walk up and use devices. The current model of requiring an app for every possible device just doesn't scale. By enabling proximate discovery through the UriBeacon standard, any number of devices can be easily found nearby. This creates an extension of the conventional Web to include the physical world, and we call this the Physical Web. Because the Web is open, the Physical Web will have all the properties that have allowed the conventional Web to grow unabated. By broadcasting simple URLs it decentralizes the entire system. There is no central server, no gatekeeper. This is unlike nearly every other commercial smart device system today.

In addition, it opens up a new type of ephemeral interaction, where any device can sprout a virtual flash card of simple information, or if necessary, more complex interaction. By making this universal so every device can read it, from smartphones, to tablets, or eventually heads-up displays, it means that everything has the potential to be smarter, and nearly any device can offer a virtual interface.

But by far the most important aspect of the Physical Web approach is that it can be built upon easily using the flexible format of the URL is to grow and adapt. Furthermore, using semantic tools like schema.org, the way IoT devices can be controlled and organized can also continue to evolve. Just as the Web has grown and evolved over the last 25 years, we expect the Physical Web to adapt to the expanding reach of the IoT for many years to come.

\section{REFERENCES}

[1] Schilit, B. N., Sengupta U., Device Ensembles, IEEE Computer, vol. 37, no.12, (Dec 2004), pp. 56-64, doi:10.1109/MC.2004.241

[2] Gartner, Newsroom, Gartner Says the Internet of Things Installed Base Will Grow to 26 Billion Units by 2020, (Dec 2013), http://www.gartner.com/newsroom/id/2636073

[3] Berners-Lee, T., Cailliau, R., Luotonen, A., Nielsen, H.F., Secret, A., The World-Wide Web, CACM, 37(1994), pp. 7682.

[4] Homes, D., The Dynamic Domain Name System (DNS) Infrastructure, http://www.f5.com, (June 2012).

[5] Heydon, R., Bluetooth Low Energy, Prentice Hall, 2013, ISBN-13: 978-0-13-288836-3.

[6] The UriBeacon Open Standard, (Apr 2015) http://uribeacon.org

[7] Want, R., Schilit, B.N., Jenson, S., Enabling the Internet of Things, IEEE Computer, vol. 48, no.1, (Jan 2015), pp. 28-35. doi: 10.1109/MC.2015.12

[8] Antonini, M., Cirani, S., Ferrari, G., Medagliani, P., Picone, M., Veltri, L., Lightweight multicast forwarding for service discovery in low-power IoT networks, IEEE Software, Telecoms. and Computer Networks (SoftCOM 2014), pp.133138, (Sept. 2014), doi: 10.1109/SOFTCOM.2014.7039103

[9] The World Wide Web Consortium, Service Worker, W3C Working Draft, http://www.w3.org/TR/service-workers/, (Feb 2025).

[10] Page, L., Brin, S., Motwani, R., Winograd, T., The PageRank Citation Ranking: Bringing Order to the Web, Stanford University, Technical Report, 1998.

[11] What is Schema.org? (March 2015), http://schema.org.

[12] Universal Plug and Play (UPnP) Device Architecture 1.1, UPnP Forum, (Oct 15 $\left.5^{\text {th }} 2008\right)$. 